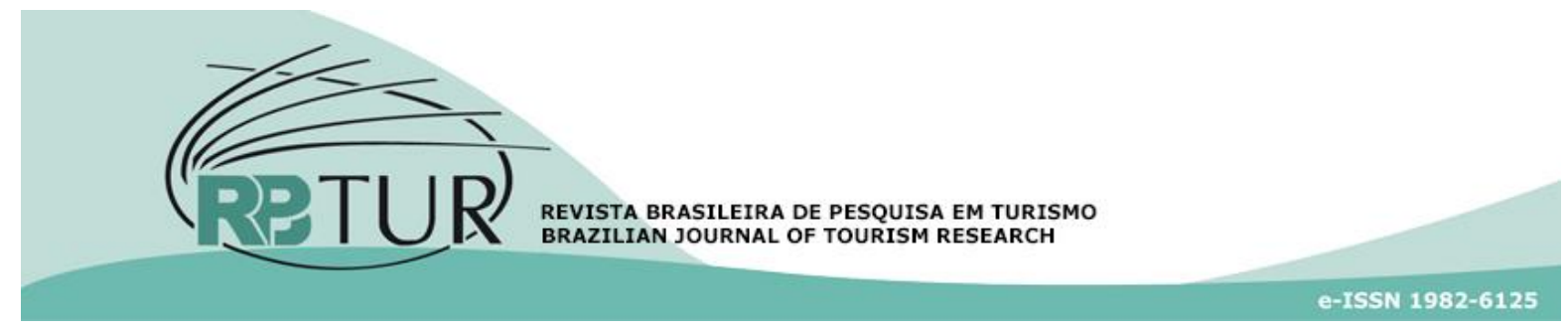

Relatório Anual 2020

\title{
Submissões recebidas
}

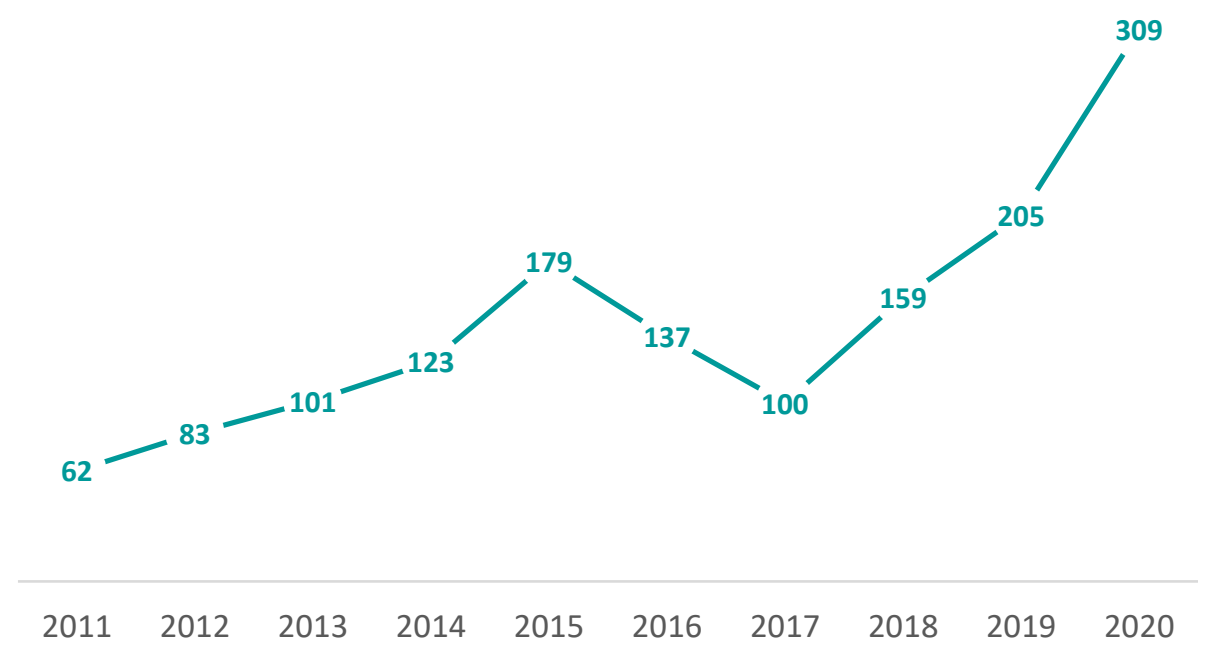

\section{Avaliadores}

1. Adriana Fumi Chim-Miki, Universidade Federal de Campina Grande, PB

2. Aguinaldo Cesar Fratucci, Universidade Federal Fluminense, RJ

3. Airton José Cavenaghi, Universidade Anhembi Morumbi, SP

4. Alexandre Augusto Biz, Universidade Federal de Santa Catarina, SC

5. Alexandre Panosso Netto, Universidade de São Paulo, SP

6. Alice Leoti, Universidade Federal do Pampa, RS

7. Alissandra Nazareth de Carvalho, Universidade Federal de São Carlos, SP

8. Almir Félix Batista de Oliveira, Universidade Federal do Rio Grande do Norte, RN

9. Altair Sancho-Pivoto, Universidade Federal de Juiz de Fora, MG

10. Amanda Arrais Mousinho, Universidade de São Paulo, SP

11. Ambrózio Correa de Queiroz Neto, Centro Federal de Educação Tecnológica Celso Suckow da Fonseca, RJ

12. André Falcão Durão, Universidade Federal de Pernambuco, PE

13. André Fontan Köhler, Universidade de São Paulo, SP

14. Andre Riani Costa Perinotto, Universidade Federal do Piauí, PI

15. Angela Teberga de Paula, Universidade Federal de Tocantins, TO

16. Bernardo Lazary Cheibub, Universidade Federal Fluminense, RJ

17. Bianca Freire Medeiros, Universidade de São Paulo, SP

18. Brenno Vitorino Costa, Instituto Federal de Educação, Ciência e Tecnologia de São Paulo, SP

19. Bruna Melo, Pontifícia Universidade Católica do Rio Grande do Sul, RS

20. Bruna Ranção Conti, Universidade Federal do Estado do Rio de Janeiro, RJ

21. Bruno Martins Augusto Gomes, Universidade Federal do Paraná, PR

22. Camila Vaz Mattos Fraga Vieira, Universidade Federal Fluminense, RJ 
23. Carlos Alberto Cioce Sampaio, Fundação Universidade Regional de Blumenau / Universidade Federal do Paraná, SC / PR

24. Carlos Eduardo de Almeida Ramoa, Universidade do Vale do Itajaí, SC

25. Carlos Eduardo Silveira, Universidade Federal do Paraná, PR

26. Carlos Peixeira Marques, Universidade de Trás-os-Montes e Alto Douro, Portugal

27. Carolina Lescura De Carvalho Castro Volta, Universidade Federal de Ouro Preto, MG

28. Carolina Todesco, Universidade Federal do Rio Grande do Norte, RN

29. Claudia Corrêa de Almeida Moraes, Universidade Federal Fluminense, RJ

30. Claudio José Stefanini, Universidade Anhembi Morumbi, SP

31. Cynthia Menezes Mello, Pesquisadora Independente,

32. Dalila Müller, Universidade Federal de Pelotas, RS

33. Dalila Rosa Hallal, Universidade Federal de Pelotas, RS

34. Dan Gabriel D'Onofre, Universidade Federal Rural do Rio de Janeiro, RJ

35. Daniel Pires Vieira, Universidade de Brasília, DF

36. Danielli Cristina Granado, Universidade Estadual Paulista Júlio de Mesquita Filho, SP

37. Davi Andrade, Universidade Federal do Maranhão / Universidade de São Paulo, MA / SP

38. David Leonardo Bouças da Silva, Universidade Federal do Maranhão, MA

39. Diomira Maria Cicci Pinto Faria, Universidade Federal de Minas Gerais, MG

40. Donária Coelho Duarte, Universidade de Brasília, DF

41. Dores Cristina Grechi, Universidade Estadual de Mato Grosso do Sul, MS

42. Dyego de Oliveira Arruda, Centro Federal de Educação Tecnológica Celso Suckow da Fonseca, RJ

43. Edar da Silva Añaña, Universidade Federal de Pelotas / Universidade Federal do Rio Grande / Universidade do Vale do Itajaí, RS / SC

44. Edegar Luis Tomazzoni, Universidade de São Paulo, SP

45. Edmur Stoppa, Universidade de São Paulo, SP

46. Eduardo Picanço Cruz, Universidade Federal Fluminense, RJ

47. Elisangela Aparecida Machado da Silva, Universidade de Brasília, DF

48. Elizabete Sayuri Kushano, Universidade Federal do Paraná, PR

49. Eloisa Pereira Barroso, Universidade de Brasília, DF

50. Eloise Silveira Botelho, Universidade Federal do Estado do Rio de Janeiro, RJ

51. Ericka Amorin, pesquisadora do GOVCOOP, Universidade de Aveiro, Portugal

52. Esdras Matheus Matias, Universidade Federal da Paraíba, PB

53. Everaldo Batista da Costa, Universidade de Brasília, DF

54. Fabia Trentin, Universidade Federal Fluminense, RJ

55. Fabricio Scarpeta Matheus, University of Northern British Columbia, Canadá

56. Fernando Luiz Araujo Sobrinho, Universidade de Brasília, DF

57. Francielle de Lima, Universidade Federal do Pampa, RS

58. Francisco Antonio Anjos, Universidade do Vale do Itajaí, SC

59. George Bedinelli Rossi, Universidade de São Paulo, SP

60. Glauria Janaina dos Santos, Instituto Federal de Educação, Ciência e Tecnologia da Bahia, BA

61. Gleice Regina Guerra, Universidade de São Paulo, SP

62. Hélio Fernando Lôbo Nogueira da Gama, Universidade Estadual de Santa Cruz, BA

63. Heros Augusto Santos Lobo, Universidade Federal de São Carlos, SP

64. Ieda Margarete Oro, Universidade do Oeste de Santa Catarina, SC

65. Isabel Baptista, Universidade Católica do Porto, Portugal

66. Isabel Jurema Grimm, Instituto Superior de Administração e Economia, PR

67. Isabela Barbosa Frederico, Universidade Estadual de Campinas, SP

68. Ivaneli Schreinert dos Santos, Universidade de São Paulo, SP

69. Izabel Cristina Augusto de Souza Faria, Universidade Federal do Estado do Rio de Janeiro, RJ

70. Izac de Oliveira Belino Bonfim, Universidade Estadual de Mato Grosso do Sul, MS

71. Jean Henrique Costa, Universidade Estadual do Rio Grande do Norte, RN

72. Jefferson Lorencini Gazoni, Universidade de Brasília, DF

73. Jennifer Caroline Soares, Universidade Federal de Sergipe, SE

74. Jessica Vieira de Souza Meira, Universidade do Vale do Itajaí, SC

75. João Paulo Faria Tasso, Universidade de Brasília, DF

76. Joice Lavandoski, Universidade Federal do Estado do Rio de Janeiro, RJ 
77. Juliana Medaglia, Universidade Federal do Paraná, PR

78. Keila Cristina Nicolau Mota, Instituto Federal de Educação, Ciência e Tecnologia do Ceará, CE

79. Kerlei Eniele Sonaglio, Universidade Federal do Rio Grande do Norte, RN

80. Kerley Santos Alves, Universidade Federal de Ouro Preto, MG

81. Kettrin Farias Bem Maracajá, Universidade Federal de Campina Grande, PB

82. Leandro Benedini Brusadin, Universidade Federal de Ouro Preto, MG

83. Leilianne Michelle Trindade da Silva Barreto, Universidade Federal do Rio Grande do Norte, RN

84. Lélio Galdino Rosa, Universidade Federal Rural do Rio de Janeiro, RJ

85. Letícia Bianca Barros de Moraes Lima, Instituto Federal de Educação, Ciência e Tecnologia de Brasília, DF

86. Lício Valério Lima Vieira, Instituto Federal de Educação, Ciência e Tecnologia de Sergipe, SE

87. Liliane Cristine Schlemer Alcântara, Universidade Federal de Mato Grosso, MT

88. Linda Maria Rodrigues, Universidade Federal do Maranhão, MA

89. Lindemberg Medeiros de Araujo, Universidade Federal de Alagoas, AL

90. Lívia Morais Garcia Lima, Universidade Estadual de Campinas, SP

91. Lucas Goulart, Universidade de São Paulo, SP

92. Luciana Araújo de Holanda, Universidade Federal de Pernambuco, PE

93. Luciana Brandão Ferreira, Universidade de São Paulo, SP

94. Luciane Scheuer, Universidade Estadual do Paraná, PR

95. Luciano Torres Tricárico, Universidade do Vale do Itajaí, SC

96. Luis Fernando Hor-Meyll, Pontifícia Universidade Católica do Rio de Janeiro / Universidade Federal do Rio de Janeiro, RJ

97. Luiz Carlos da Silva Flores, Universidade do Vale do Itajaí, SC

98. Luiz Gonzaga Godoi Trigo, Universidade de São Paulo, SP

99. Luiz Mendes Filho, Universidade Federal do Rio Grande do Norte, RN

100. Marcelino Castillo Nechar, Universidad Autónoma del Estado de México, México

101. Marcelo Chiarelli Milito, Universidade Federal do Rio Grande do Norte, RN

102. Marcelo da Silva Taveira, Universidade Federal do Rio Grande do Norte, RN

103. Marcelo Nunes, Universidade Federal do Rio de Janeiro, RJ

104. Márcia Maria Cappellano dos Santos, Universidade de Caxias do Sul, RS

105. Marcia Zampieri Grohmann, Universidade Federal de Santa Maria, RS

106. Marcio Luiz Marietto, Instituto Politécnico de Leiria / Universidade Regional Integrada do Alto Uruguai e das Missões, Portugal / RS

107. Márcio Marreiro das Chagas, Instituto Federal de Educação, Ciência e Tecnologia do Rio Grande do Norte, RN

108. Marcio Nakayama Miura, Universidade Estadual do Paraná, PR

109. Marco Aurelio Avila, Universidade Estadual de Santa Cruz, BA

110. Marconi Costa, Universidade Federal de Pernambuco, PE

111. Marcos Aurelio Tarlombani Silveira, Universidade Federal do Paraná, PR

112. Marcos Eduardo Carvalho Gonçalves Knupp, Universidade Federal de Ouro Preto, MG

113. Marcos Luiz Filippim, Universidade Federal do Paraná, PR

114. Margarete Araujo Teles, Universidade Federal do Paraná, PR

115. Maria Angela de Abreu Cabianca, Universidade Anhembi Morumbi, SP

116. Maria Goretti da Costa Tavares, Universidade Federal do Pará, PA

117. Maria Henriqueta Sperandio Garcia Gimenes Minasse, Universidade Anhembi Morumbi, SP

118. Maria Luiza Cardinale Baptista, Universidade de Caxias do Sul / Universidade Federal do Amazonas, RS / AM

119. Mariana Brandâo, Fundação Getúlio Vargas (RJ), RJ

120. Mariana Bueno de Andrade-Matos, Universidade de São Paulo, SP

121. Mariana de Freitas Coelho, Universidade Federal de Minas Gerais, MG

122. Marília Gomes dos Reis Ansarah, Universidade Paulista, SP

123. Marina Monteiro da Silva, Universidade de São Paulo, SP

124. Marinês Walkowski, Associação Acolhida na Colônia / Secretaria da Indústria, Comércio e

Turismo de Urubici, SC

125. Marklea da Cunha Ferst, Universidade do Estado do Amazonas, AM

126. Marlei Salete Mecca, Universidade de Caxias do Sul, RS

127. Marlusa de Sevilha Gosling, Universidade Federal de Minas Gerais, MG 
128. Marutschka Martini Moesch, Universidade de Brasília, DF

129. Marvin Uehara, Singapore University of Social Sciences, Singapura

130. Matheus Belucio, Universidade de Coimbra, Portugal

131. Melise de Lima Pereira, Universidade Federal do Rio Grande, RS

132. Miguel Moital, Bournemouth University, Reino Unido

133. Milena Manhães Rodrigues, Universidade de São Paulo, SP

134. Mirian Rejowski, Universidade Anhembi Morumbi, SP

135. Moabe Breno Ferreira Costa, Universidade Federal do Rio Grande do Norte, RN

136. Moisés Diniz Vassallo, Universidade Federal de Itajubá, MG

137. Natalia Porto, Universidad Nacional de La Plata, Argentina

138. Natália Tavares Azevedo, Universidade Federal do Paraná, PR

139. Pablo Flores Limberger, Universidade do Vale do Itajaí, SC

140. Patrícia Zaczuk Bassinello, Universidade Federal de Mato Grosso do Sul, MS

141. Patrinês Aparecida França Zonatto, Universidade do Vale do Itajaí, SC

142. Paulo dos Santos Pires, Universidade do Vale do Itajaí, SC

143. Paulo Henrique Assis Feitosa, Universidade de São Paulo, SP

144. Pedro de Alcântara Bittencourt César, Universidade de Caxias do Sul, RS

145. Rafaela Almeida Cordeiro, ESPM, SP

146. Rafaela Camara Malerba, Instituto Federal de Educação, Ciência e Tecnologia de São Paulo / Universidade de Aveiro, SP / Portugal

147. Renata Silva Santos Camargo, Instituto Federal de Educação, Ciência e Tecnologia do Sudeste de Minas, MG

148. Ricardo de Oliveira Rezende, Ministério da Saúde, DF

149. Ricardo Eustáquio Fonseca Filho, Universidade Federal de Ouro Preto, MG

150. Roberto Marin Viestel, Instituto Federal de Educação, Ciência e Tecnologia do Sul de Minas, MG

151. Roberto Meurer, Universidade Federal de Santa Catarina, SC

152. Roberto Pessoa de Queiroz Falcão, Universidade Federal Fluminense, RJ

153. Rodrigo Amado Santos, Universidade Federal Rural do Rio de Janeiro, RJ

154. Rodrigo Corrêa D Peixoto, Universidade Federal do Pará, PA

155. Rodrigo Ladeira, Universidade Federal da Bahia, BA

156. Rodrigo Meira Martoni, Universidade Federal de Ouro Preto, MG

157. Rômulo Duarte, Universidade de São Paulo, SP

158. Sandro Alves de Medeiros, Universidade Federal de Alagoas, AL

159. Sandro Campos Neves, Universidade Federal do Paraná, PR

160. Saulo Ribeiro dos Santos, Universidade Federal do Maranhão, MA

161. Sênia Bastos, Universidade Anhembi Morumbi, SP

162. Sérgio Rodrigues Leal, Universidade Federal de Pernambuco, PE

163. Sidnei Raimundo, Universidade de São Paulo, SP

164. Silvana Pirillo Ramos, Universidade Federal de Alagoas, AL

165. Silvio Lima Figueiredo, Universidade Federal do Pará, PA

166. Silvio Luiz Gonçalves Vianna, Universidade de Caxias do Sul, RS

167. Stefânia Ordovás de Almeida, Pontifícia Universidade Católica do Rio Grande do Sul, RS

168. Stela Cristina Hott Corrêa, Universidade Federal de Minas Gerais, MG

169. Susana de Araujo Gastal, Universidade de Caxias do Sul, RS

170. Telma Bittencourt Bassetti, Universidade Federal do Estado do Rio de Janeiro, RJ

171. Teresa Cristina Catramby, Universidade Federal Rural do Rio de Janeiro, RJ

172. Thays Cristina Domareski Ruiz, Universidade do Vale do Itajaí, SC

173. Thiago Allis, Universidade de São Paulo, SP

174. Thiago do Val Simardi Beraldo Souza, ICMBio, DF

175. Thiago Duarte Pimentel, Universidade Federal de Juiz de Fora, MG

176. Tiago Savi Mondo, Instituto Federal de Educação, Ciência e Tecnologia de Santa Catarina, SC

177. Valeria de Meira Albach, Universidade Estadual de Ponta Grossa, PR

178. Valquíria Padilha, Universidade de São Paulo, SP

179. Vander Valduga, Universidade Federal do Paraná, PR

180. Vânia Lúcia Quadros Nascimento, Universidade Federal do Pará, PA

181. Vera Lucia Bogea Borges, Universidade Federal do Estado do Rio de Janeiro, RJ

182. Verônica Feder Mayer, Universidade Federal Fluminense, RJ 
183. Virginia Aparecida Castro, Universidade de São Paulo, SP

184. Vítor Silva Freire, Universidade de São Paulo, SP

185. Viviane Santos Salazar, Universidade Federal de Pernambuco, PE

186. Viviane Silva Souza, Universidade Federal Rural de Pernambuco, PE

187. Wilker Ricardo de Mendonça Nóbrega, Universidade Federal do Rio Grande do Norte, RN 